\title{
On the structural and electrical properties of modified $\mathrm{PbTiO}_{3}$ ceramics ${ }^{\dagger}$
}

\author{
$\mathrm{K}$ PRASAD*, R N P CHOUDHARY ${ }^{+}, \mathrm{S} \mathrm{N} \mathrm{CHOUDHARY}^{++}$and \\ $\mathrm{R} \mathrm{SATI}^{++}$ \\ Department of Physics, Sant Longowal Institute of Engineering and Technology, Longowal, \\ Sangrur 148 106, India \\ ${ }^{+}$Department of Physics, Indian Institute of Technology, Kharagpur 721 302, India \\ ${ }^{++}$University Department of Physics, Bhagalpur University, Bhagalpur 812007, India
}

\begin{abstract}
Ceramics $\left(\mathrm{Pb}_{0.75} \mathrm{Ca}_{0.25}\right)\left[\left(\mathrm{Ni}_{1 / 2} \mathrm{~W}_{1 / 2}\right)_{y} \mathrm{Ti}_{1-y}\right] \mathrm{O}_{3}$ for $y=0 \cdot 05,0 \cdot 10,0 \cdot 15$ and $0 \cdot 30$ have been prepared using high temperature solid state reaction method. X-ray analyses show that these materials are of single-phase perovskite type tetragonal structure. To solve inaccuracy in finding para-ferroelectric phase transition temperature $T_{\mathrm{c}}\left(\varepsilon_{\max }\right)$, we have carried out the analyses of the data using a computer program. The role of $\mathrm{Ni}$ and $\mathrm{W}$ as modifiers on structural and electrical (dielectric and pyroelectric) properties is discussed in terms of the usefulness of these materials. Results indicate that ceramic $\mathrm{Pb}_{0.75} \mathrm{Ca}_{0.25} \mathrm{Ni}_{0.025} \mathrm{~W}_{0.025^{-}}$ $\mathrm{Ti}_{0.95} \mathrm{O}_{3}$ is a good candidate for pyroelectric applications.
\end{abstract}

Keywords. Ferroelectrics; dielectric constant; pyroelectric coefficient; diffuse phase transition: lead titanate ceramics.

\section{Introduction}

Pyroelectric infrared sensor has the advantage of wavelength independent sensitivity at room temperature operation. Thus it is expected to provide various thermal detectors for the object at near room temperature which can find their use in various fields such as industrial robots, diagnosis, environmental observation, etc. Lead titanate $\left(\mathrm{PbTiO}_{3}\right.$ : $\mathrm{ABO}_{3}$ type) is a suitable material for pyroelectric infrared sensor, because it has a large pyroelectric coefficient, $\gamma$ and small dielectric constant, $\varepsilon$. Therefore it has a large figure of merit. $\mathrm{PbTiO}_{3}$ has also small temperature coefficient of $\varepsilon$ and $\gamma$ over a considerable range of working temperature because of its high Curie temperature $T_{\mathrm{c}}$.

$\left(\mathrm{Pb}_{1-x} \mathrm{Ca}_{x}\right)\left[\left(\mathrm{M}_{1 / 2} \mathrm{~W}_{1 / 2}\right)_{y} \mathrm{Ti}_{1-y}\right] \mathrm{O}_{3}$ ceramics, prepared by replacing a part of $\mathrm{Pb}^{2+}$ by $\mathrm{Ca}^{2+}$ and $\mathrm{Ti}^{4+}$ by $\mathrm{M}\left(\equiv \mathrm{Co}^{2+}\right.$ (Ichinose 1985; Mendiola et al 1989; Nadoliisky et al 1991), $\mathrm{Mn}^{2+}$ (Prasad et al 1993a, b, 1994a) or $\mathrm{Ni}^{2+}$ (Prasad et al 1994b, 1995b)) and $\mathrm{W}^{6+}$ from $\mathrm{PbTiO}_{3}$, is expected to be an excellent pyroelectric material because it is possible to control its $T_{\mathrm{c}}$ by changing the concentration of the substituents at $\mathrm{Pb}$ or Ti sites.

The phase transition of ferroelectric ceramics is smeared as a consequence of chemical composition, microstructure and sintering processes. The transition region extends over some tens of degrees around the temperature of maximum $\varepsilon\left(\varepsilon_{\max }\right)$. The electrical polarization, $P_{\mathrm{r}}$, is continuously decreasing with temperature and it is difficult to determine, with accuracy, the temperature for $P_{\mathrm{r}}=0$.

In classical perovskite type ferroelectrics, the anomalies which take place at a ferroparaelectric phase transition are generally described by the susceptibility, $\chi$, and the

\footnotetext{
*For correspondence

${ }^{\dagger}$ Paper presented at the poster session of MRSI AGM VI, Kharagpur, 1995
} 
order parameter, $P$, by the expression:

$$
\chi_{\mathrm{ij}}^{-1}=\chi_{0}^{-1}+\Sigma g_{\mathrm{ijk} 1} P_{\mathrm{k}} P_{1}
$$

The coupling constant $g_{\mathrm{ijk} \mathbf{l}}$ describes the breaking of symmetry by the phase transition (Krisch et al 1986; Jimenez et al 1987).

However, there is a set of anomalies in the behaviour of some perovskite type ferroelectrics with a diffuse phase transition (DPT) which are difficult to explain on the basis of classical theories of ferroelectrics (Jimenez et al 1987). Above the transition temperature, parameters such as refractive index (Burns and Dacol 1985), specific heat (Fouskova et al 1981), thermal expansion (Kirsch et al 1986), etc deviate from their normal behaviour in a temperature interval of some tens of degrees above $T_{\mathrm{c}}$. These anomalies have been explained by Burns and Dacol (1985) assuming the existence of local polarization which disappears at temperature much higher than $T_{\mathrm{c}}$.

The maxima of dielectric constant in DPTs are not well defined. It is thus experimentally difficult to know, with accuracy, the temperature of the ferro-paraelectric phase transition, $T_{c}$. In the paraelectric phase, dielectric constant behaves as;

$$
\varepsilon^{-1}=\varepsilon_{\max }^{-1}+A\left(T-T_{\mathrm{c}}\right)^{\Gamma},
$$

where $\Gamma$ is the diffusivity parameter - a measure of broadness in DPT, varies between 1. normal Curie-Weiss type and 2. typical diffuse transition type (Kuwata et al 1982; Alemany et al 1987) and $A$ is a constant.

In this report we have studied the role of $\mathrm{Ni}$ and $\mathrm{W}$ as a modifier on structural and electrical (dielectric and pyroelectric) properties of $\left(\mathrm{Pb}_{0.75} \mathrm{Ca}_{0.25}\right)\left[\left(\mathrm{Ni}_{1 / 2} \mathrm{~W}_{1 / 2}\right)_{y^{-}}\right.$ $\left.\mathrm{Ti}_{1-y}\right] \mathrm{O}_{3}$ ceramics for $y=0.05,0.10,0.15$ and 0.30 . Computer fitting has been done in order to obtain the value of $T_{\mathrm{c}}$ using $\varepsilon(T)$ data, which gives the best agreement between experimental and expression (2). Also, the experimental results of dielectric constant, in the present ceramics, has been discussed by means of Burns and Dacol's assumptions.

\section{Experimental}

The stoichiometric mixtures of $\left(\mathrm{Pb}_{0.75} \mathrm{Ca}_{0.25}\right)\left[\left(\mathrm{Ni}_{1 / 2} \mathrm{~W}_{1 / 2}\right)_{y} \mathrm{Ti}_{1-y}\right] \mathrm{O}_{3}$ for $y=0.05$, $0 \cdot 10,0 \cdot 15$ and $0 \cdot 30$ were prepared through the process shown in figure 1 , starting from high purity $(99.9 \%)$ oxides. The obtained material is dried and thermally treated at $1020^{\circ} \mathrm{C}$ for $4 \mathrm{~h}$. From these materials a set of thin pellets of $12.5 \mathrm{~mm}$ diameter and $1.5 \mathrm{~mm}$ height were prepared under isostatic pressure of $6 \times 10^{7} \mathrm{Kg} \mathrm{m}^{-2}$. Once pressed, the pellets were fired at $1100^{\circ} \mathrm{C}$ and times between $0.5 \mathrm{~h}$ to $4 \mathrm{~h}$. The synthesis process were monitored by XRD-technique.

For preliminary structural studies, X-ray diffractograms (XRD) were recorded at room temperature by X-ray diffractometer PHILIPS (PW 1710-Holland) using nickel filtered $\mathrm{CuK} \alpha$-radiation $(\lambda=1.5418 \AA)$ at a scanning rate of $2^{\circ}(2 \theta) / \mathrm{min}$. The angular range $2 \theta$ covered was $10^{\circ}-70^{\circ}$. To measure $\varepsilon$ and loss $(\tan \delta)$ of the compounds, air drying silver paint was applied on both the large faces of the samples to serve as electrodes. Measurements of $\varepsilon$ and $\tan \delta$ were carried out as a function of temperature $\left(26^{\circ} \mathrm{C}-300^{\circ} \mathrm{C}\right)$ with a.c. field of $10 \mathrm{KHz}$ frequency using LCR Hi-Tester(HIOKI-Japan) and with GenRad 1620 AP Capacitance measuring assembly, USA. The temperature of 


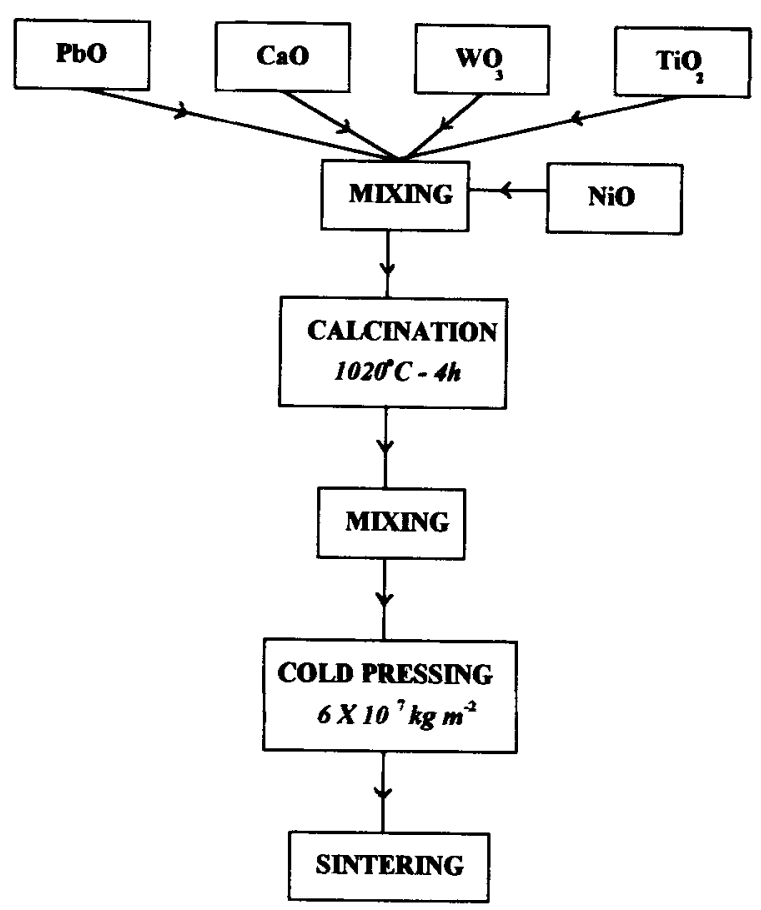

Figure 1. Flow chart for preparation of the materials.

the samples was varied at intervals of $2^{\circ} \mathrm{C} / \mathrm{min}$ using a furnace built for this purpose and a temperature controller (Indotherm 401D). The temperature was measured with a chromel-alumel thermocouple (accuracy $\pm 0.25 \%$ ). To overcome the moisture effect on the electrical properties, the samples were preheated to $100^{\circ} \mathrm{C}$ to evaporate the moisture and then cooled to room temperature and then experiments were carried out. Differential scanning calorimeter (DSC) curve was obtained by a computer-controlled differential scanning calorimeter (Model DSC-4) of Perkin-Elmer, USA. The experiments were carried out between room temperature $\left(26^{\circ} \mathrm{C}\right)$ to $450^{\circ} \mathrm{C}$ in nitrogen atmosphere at a heating rate of $20^{\circ} \mathrm{C} / \mathrm{min}$. Alumina crucibles were used in the experiment.

\section{Fitting procedure}

To solve the inaccuracy in finding $T_{\mathrm{c}}\left(\varepsilon_{\max }\right)$ we first took $T_{\mathrm{c}}$ as the mean value of the temperature at the extrema (extended on a $10^{\circ} \mathrm{C}$ interval, where $\varepsilon$ had an apparently constant value) of the interval and fitted to expression (2) by the method of least squares. Also, in order to optimize the regression coefficient $R^{2}$ between (2) and experimental data, we took values of $T_{\mathrm{c}}$ in a $\pm 4^{\circ} \mathrm{C}$ interval around the previous mean value. The optimized results are shown in table 1. 
Table 1. Statistical analysis for diffuse phase transition and some materials parameters.

\begin{tabular}{lrrrrrrrrr}
\hline$y$ & $c / a$ & $\varepsilon_{\mathrm{RT}}$ & $\varepsilon_{\max }$ & $\begin{array}{c}\tan \delta \\
\left(\times 10^{3}\right)\end{array}$ & $\left.T_{\mathrm{C}}{ }^{\circ} \mathrm{C}\right)$ & $\Gamma$ & $\begin{array}{r}\text { No. of } \\
\text { points }\end{array}$ & $\begin{array}{l}\text { Regr. } \\
\text { coeff. } \\
\left(\boldsymbol{R}^{2}\right)\end{array}$ \\
\hline 0.05 & 1.045 & 166 & 4751 & 3.0 & 253 & 1.21 & 59 & 0.9967 \\
0.10 & 1.041 & 181 & 2987 & 3.8 & 241 & 1.28 & 80 & 0.9928 \\
0.15 & 1.037 & 192 & 1648 & 5.0 & 233 & 1.43 & 84 & 0.9979 \\
0.30 & 1.032 & 209 & 753 & 6.7 & 197 & 1.52 & 102 & 0.9987 \\
\hline
\end{tabular}

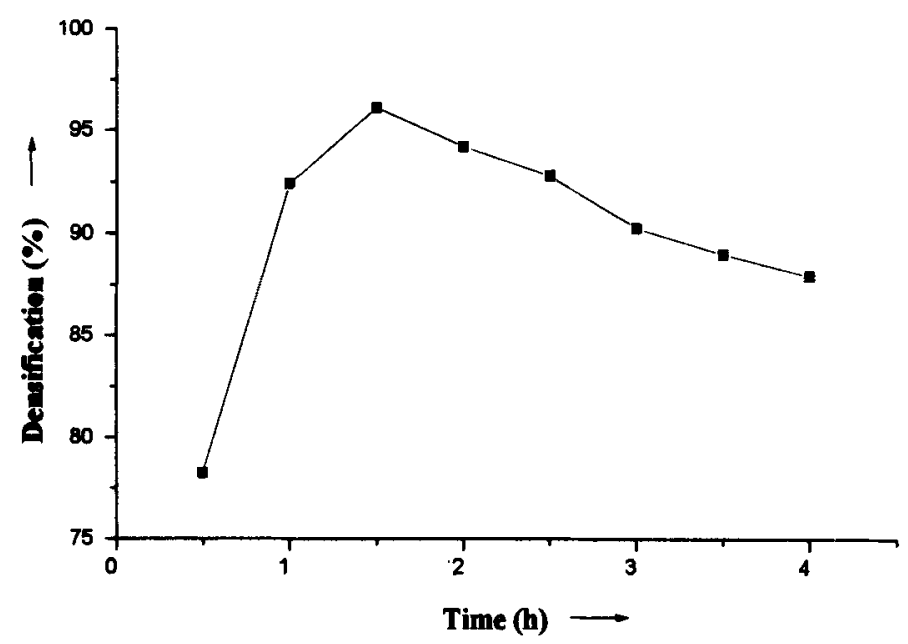

Figure 2. Variation of densification (\%) with sintering time (h) for $\mathrm{Pb}_{0.75} \mathrm{Ca}_{0.25} \mathrm{Ni}_{0.025}$ $\mathrm{W}_{0.025} \mathrm{Ti}_{0.95} \mathrm{O}_{3}$ at $1100^{\circ} \mathrm{C}$.

\section{Results and discussion}

Figure 2 shows the densification of the pellets as a function of sintering time for $\mathrm{Pb}_{0.75} \mathrm{Ca}_{0.25} \mathrm{Ni}_{0.025} \mathrm{~W}_{0.025} \mathrm{Ti}_{0.95} \mathrm{O}_{3}$ ceramic. The first part of the isothermal curve $(0.5$ to $1.5 \mathrm{~h})$ can be explained on the basis of the consideration that the sintering mechanisms dominate the process and a recovery of densification is observed. For higher times a degradation of the ceramic takes place. $96 \%$ theoretical density is achieved for the ceramics sintered at $1100^{\circ} \mathrm{C}$ for $1.5 \mathrm{~h}$. The sintered density decreases due to the evaporation of $\mathrm{PbO}$ as the sintering time increases.

All samples are found to have single phase perovskite-type tetragonal structure $(c / a<1.063)$ from the XRD analyses. Also, with the increment in $\mathrm{Ni}$ and $\mathrm{W}$ content, $c / a$ decreases (table 1).

Figure 3 shows the temperature dependence of $\varepsilon$ for the present ceramics. As typical of normal ferroelectrics, $\varepsilon$ increases gradually with increment in temperature up to $T_{\mathrm{c}}$ and then decreases. A distinct deviation from Curie-Weiss law in paraelectric phase, can be observed in figures 3 and 4, and follows expression (2). To see if the transition has a diffusive character, computer fitting, to expression (2), has been done. We found $\Gamma>1$ for all cases (table 1), which confirms the diffusive character of phase transition 


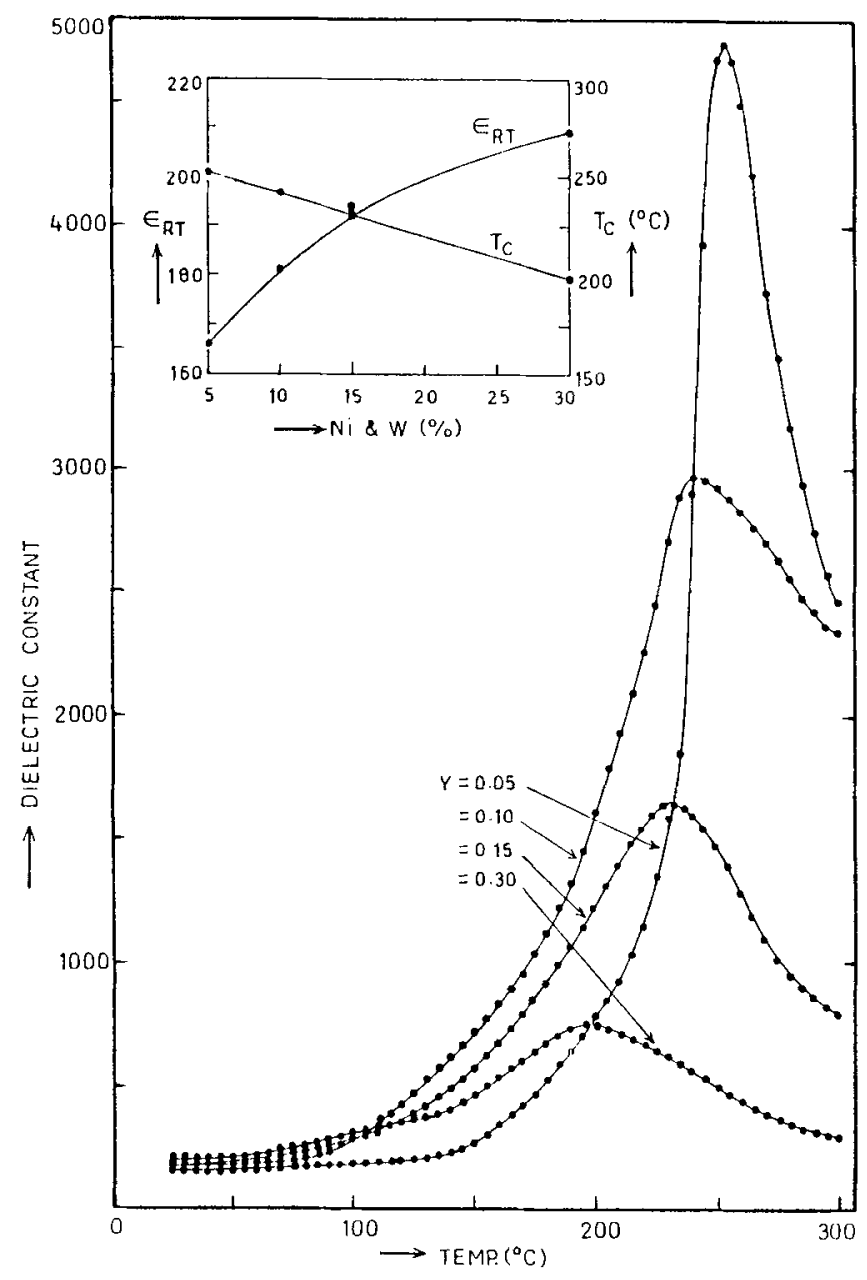

Figure 3. Temperature variation of $\varepsilon$ of $\left(\mathrm{Pb}_{0.75} \mathrm{Ca}_{0.25}\right)\left[\left(\mathrm{Ni}_{1,2} \mathrm{~W}_{1 / 2}\right)_{1} \mathrm{~T}_{1-1}\right] \mathrm{O}_{3}$ at $10 \mathrm{kHz}$.

(del Olmo et al 1987). This may be due to the presence of more than one cation in the sublattice, that should produce some kind of heterogeneities (Mendiola et al 1989; Prasad et al 1995a). The behaviour of $\varepsilon$ with temperature above $T_{\mathrm{c}}$ may be due to the electrical conductivity of the materials that modifies the value of the capacitance as the temperature increases.

We find that with the increment in $\mathrm{Ni}$ and $\mathrm{W}$ content $T_{\mathrm{c}}$ decreases, $\varepsilon_{\mathrm{RT}}$ increases (inset figure 3 ) while dielectric peak $\left(\varepsilon_{\max }\right)$ decreases and shifts towards lower temperature side and $\varepsilon-T$ curve flattens (figure 3 ). The decrease in $\varepsilon_{\max }$ implies that the substitution of $\mathrm{Ni}$ and $\mathrm{W}$ ions reduces the dipole moment of the lattice and lowers the peak dielectric constant. Temperature coefficient of dielectric constant is defined as $T_{\mathrm{C} \varepsilon}=\left(\varepsilon_{\mathrm{T}}-\varepsilon_{\mathrm{RT}}\right)$ / $\varepsilon_{\mathrm{RT}} \times 100$ (Halliyal et al 1987; Prasad et al 1995a). A very small variation in $T_{\mathrm{C} \varepsilon}$ with temperature has been found over a considerable range of working temperature which is very much desirable for pyroelectric application. $\left.T_{\mathrm{C}_{\varepsilon}}\right|_{100 \mathrm{C}}=4.22 \%$ has been found for $y=0.05$ which is also clear from figure 3 that $\varepsilon$ seems almost independent of temperature up to $100^{\circ} \mathrm{C}$. In all the materials, $\tan \delta$ was found to be $\sim 10^{-2}$. The low $\tan \delta$ of this 


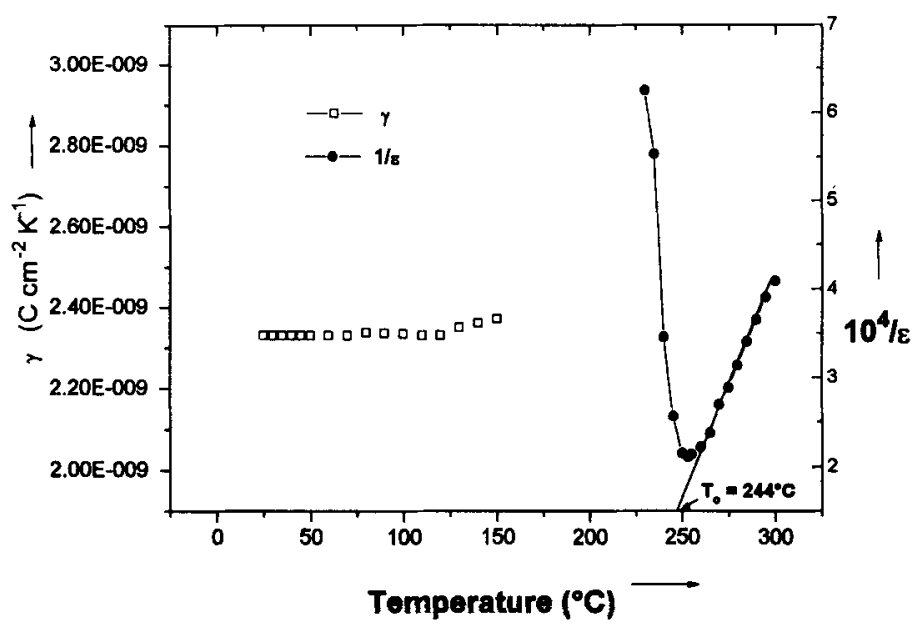

Figure 4. Temperature variation of dielectric stiffness $(1 / \varepsilon)$ and pyroelectric coefficient $(\gamma)$ of $\mathrm{Pb}_{0.75} \mathrm{Ca}_{0.25} \mathrm{Ni}_{0.025} \mathrm{~W}_{0.025} \mathrm{Ti}_{0.95} \mathrm{O}_{3}$.

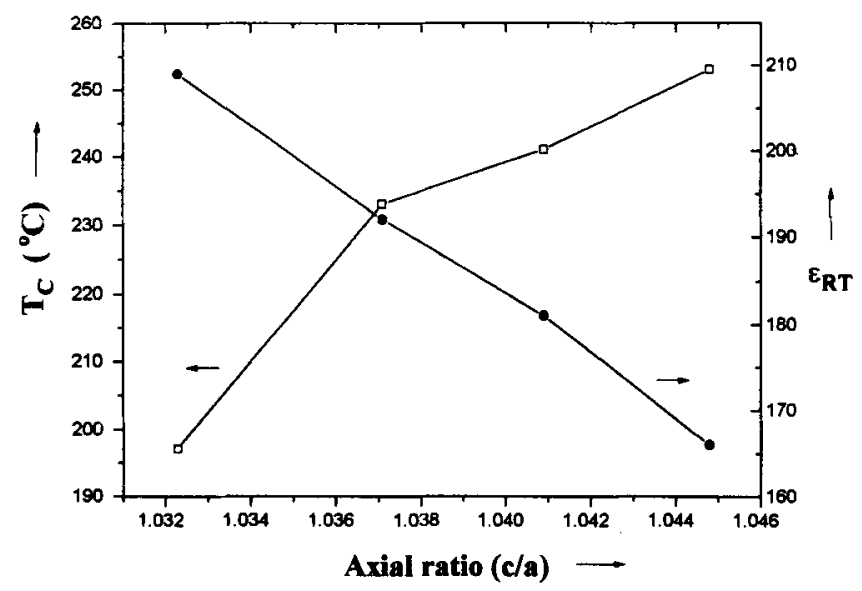

Figure 5. Variation of $T_{\mathrm{C}}$ and $\varepsilon_{\mathrm{RT}}$ with axial ratio $(c / a)$.

kind can be advantageous when improved detectivity is required. By taking reciprocal of $\varepsilon$ (figure 4) the $T_{0}$ (Curie-Weiss point) temperature is extrapolated. It is found that $T_{0}<T_{\mathrm{C}}$ for all materials, which confirms the phase transition to be of the first order. Linear fitting of the results allow us to obtain the Curie-Weiss constant $\mathrm{C}$. The value of $\mathrm{C}$ has been estimated to be $\sim 10^{5 \circ} \mathrm{K}$ for the present specimens.

Assuming that the ferroelectricity in the present materials is only due to dipolar contribution, then we have

$$
\varepsilon=\frac{T-\mathrm{C}}{T-T_{\mathrm{C}}},
$$

where $\mathrm{C}=2 N \mu^{2} / 9 \varepsilon_{0} k_{\mathrm{B}}, T_{\mathrm{C}}=N \mu^{2} / 9 \varepsilon_{0} k_{\mathrm{B}}, N$ is the number of molecules per unit volume, $k_{\mathrm{B}}$ the Boltzmann constant and $\mu$ the permanent dipole moment. Thus, as 
$T \rightarrow T_{\mathrm{C}}$, from (3) $\varepsilon \rightarrow \infty$. This divergence is called 'polarization catastrophe'. From this it follows that at $T<T_{\mathrm{C}}$ there should be spontaneous polarization and material should be ferroelectric. Further, we have $\mathrm{C}=2 T_{\mathrm{C}}$. But experimental results show that $\mathrm{C} \gg 2 T_{\mathrm{C}}$ and are of the order of $10^{5 \circ} \mathrm{K}$. Hence, ferroelectricity in the present modified $\mathrm{PbTiO}_{3}$ ceramics is not just be due to permanent dipole moments but significant contribution must come from relative displacement of all $\mathrm{B}-\mathrm{O}$ ions in a cooperative manner (Harada et al 1970; Srivastava and Srinivasan 1991). This happens due to hybridization between the $\mathrm{B}$ ( Ti $3 d$ states) cation and $\mathrm{O}(2 p$ states) that essentially weakens the short-range repulsions and allow the ferroelectric transition (Cohen 1992).

The relation between $T_{\mathrm{C}}$ and $\varepsilon_{\mathrm{RT}}$ with axial ratio $(c / a)$ is shown in figure 5 . The curve for $T_{\mathrm{C}}$ shows a positive slope. The results indicated the lowering of $T_{\mathrm{C}}$ and increase in $\varepsilon_{\mathrm{RT}}$ with the decrease of tetragonal distortion (Prasad et al 1995b). This can be explained on the basis that the delicate balance of short-range forces (favouring the non-polar cubic phase) and long-range Coulomb forces (favouring the ferroelectric state) makes the transition sensitive to defects (substitutions) that modify the shortrange interactions and to carriers (e.g. photoelectrons) that screen the long-range field (Cohen 1992).

Pyroelectric currents in the unpoled state were measured using direct measurement technique (Bayer and Roundy 1972). Only the ceramic for $y=0.05$ exhibited pyroelectric effect. The $\gamma$ is found to be almost temperature independent from room temperature to $100^{\circ} \mathrm{C}$ (figure 4 ) which is essential for pyroelectric applications. The value of pyroelectric coefficient at room temperature has been found to be

$$
\gamma_{\mathrm{RT}}\left(=I_{\mathrm{PE}} / A(\mathrm{~d} T / \mathrm{d} t)\right)=2 \cdot 32 \times 10^{-9} \mathrm{C} \mathrm{cm}^{-2} K^{-1}
$$

where $I_{\mathrm{PE}}$ and $A$ are the pyroelectric current and surface area of the sample respectively. The appropriate 'figures of merit' for application of interest $F_{\mathrm{I}}\left(=\gamma / \rho \mathrm{C}_{\mathrm{p}}\right), F_{\mathrm{V}}\left(=\gamma / \rho \mathrm{C}_{\mathrm{p}} \varepsilon\right)$ and $F_{\mathrm{D}}\left(=\gamma / \rho \mathrm{C}_{\mathrm{p}} \sqrt{\varepsilon \tan \delta}\right)$, where $\mathrm{C}_{\mathrm{p}}$ is specific heat (Prasad et al 1995b) were calculated. The values of which are, respectively, $9.10 \times 10^{-10}, 3.98 \times 10^{-12}$ and $4.74 \times 10^{-10}$. Reasonably high values of figures of merit make $\mathrm{Pb}_{0.75} \mathrm{Ca}_{0.25} \mathrm{Ni}_{0.025}$ $\mathrm{W}_{0.025} \mathrm{Ti}_{0.95} \mathrm{O}_{3}$ ceramic promising for pyroelectric device applications. Moreover, these materials have reproducible properties, they are suitable for mass production and these oxide ceramics are easy to make in the form of thin specimens for efficient pyroelectric detection; and it may not be necessary to pole these materials after fabrication.

\section{References}

Alemany C, Gallo J G, Jimenez B, Maurer E and Mendiola J 1984 Ferroelectrics 54137

Bayer R L and Roundy C B 1972 Ferroelectrics 3333

Burns G and Dacol F H 1985 J. Appl. Phys. Japan Suppl. 2485

Cohen Ronald E 1992 Nature 358136

del Olmo L, Pardo L, Pina J I, Fandiño C, Alemany C, Mendiola J, Jimenez B and Maurer E 1987 Patente

Española de Invenciòn \# 8603556

Fouskova A, Kohl V, Krainik N N and Mylnilova I E 1981 Ferroelectrics 34119

Halliyal A, Kumar U, Newnham R E and Cross L E 1987 J. Am. Ceram. Soc. 70119

Harada J, Pedersen T and Barnea Z 1970 Acta Crystallogr. A26 608

Ichinose N 1985 J. Am. Ceram. Soc. Bull. 641581

Jimenez B, Frutos J de and Alemany C 1987 J. Phys. Chem. Solids 48877 
Kirsch B, Schmitt H and Müser H E 1986 Ferroelectrics 68275

Kuwata J, Uchino K and Nomura S 1982 Jpn J. Appl. Phys. 211298

Mendiola J, Jimenez B, Alemany C, Pardo L and del Olmo L 1989 Ferroelectrics 94183

Nadoliisky M M, Vassileva T K and Yanehev R V 1991 Ferroelectrics 118111

Prasad K, Choudhary S N, Choudhary R N P and Yadav K L 1993a J. Mater. Sci. Lett. 12758

Prasad K, Sati R, Choudhary R N P and Sinha T P 1993b Bull. Mater. Sci. 16679

Prasad K, Singh N P, Choudhary R N P and Sati R 1994a Indian J. Pure \& Appl. Phys. 32764

Prasad K, Sati R, Choudhary R N P and Singh N P 1994b Phys. Status Solidi 143a 423

Prasad K, Choudhary R N P and Sati R 1995a Proc. Indian Nat. Sci. Acad. Part A 61337

Prasad K, Choudhary R N P and Sati R 1995b Nat. Acad. Sci. Lett. 18145

Srivastava C M and Srinivasan C 1991 Science of engineering materials (New Delhi: Wiley Eastern Ltd.) 